\title{
Research Note \\ The evolutionary status of the early-type binary HD 115071
}

\author{
C. Lloyd and D. J. Stickland
}

Space Science \& Technology Department, Rutherford Appleton Laboratory, Chilton, Didcot, Oxon. OX11 0QX, UK

Received 22 December 2000 / Accepted 2 March 2001

\begin{abstract}
An analysis of the Hipparcos photometry of HD 115071 shows that it is an ellipsoidal variable with a period of 2.73126 days, twice that suggested previously. Modelling the light curve combined with the available radial velocities suggests that the more massive, hotter component is smaller and less luminous than the cooler component, which is probably filling its Roche lobe. The likely age of the system indicates that it is a MS + MS blue straggler, created by Case A mass exchange.
\end{abstract}

Key words. stars: binaries: spectroscopic - stars: blue stragglers - stars: early-type - stars: evolution stars: variable: general

\section{Introduction}

HD 115071 (HIP 65747, LSS 2998, O9 Vn, $V=7.94$ ) is one of the brightest stars in a complex region of star formation containing the $\mathrm{H}$ II region RCW 75 and the Cen R1 association, and lies approximately $2^{\circ}$ east of the Cen OB1 association, at a distance of $\sim 2 \mathrm{kpc}$. The RCW 75 complex is a small feature on the western edge of a large molecular cloud and region of high dust extinction. At the heart of the $\mathrm{H}$ II region lies the small, sparse open cluster Stock 16, which is closely associated with a pinched off section of an "elephant's trunk" feature of high obscuration. Stock 16 has an age of 3-5 Myr (Turner 1985) and the brightest star in the cluster, HD 115455, O7.5 III((f)) (LSS 3019), is evolving away from the main sequence. The region has seen several generations of star formation and, although the production of massive stars has now ceased, the residue of the last round can probably be seen in lowmass pre-main-sequence stars on the western edge of the H II region (Turner 1985). Stock 16 is unusual in that it contains a very high proportion of binaries amongst its brightest stars (see Turner 1985 for discussion), and this provides further indirect evidence that the cluster, while young, is not very young.

HD 115071 lies some $15^{\prime}$ west of Stock 16, towards Cen OB1, against a background of more distant stars.

Send offprint requests to: C. Lloyd,

e-mail: cl@ast.star.rl.ac.uk
HD 115071 is regarded as part of the wider population of Cen OB1 although its spatial separation and distance modulus $V_{0}-M_{V}=11.13$ place it rather closer to the Stock 16/RCW 75 complex than Cen OB1 $\left(V_{0}-M_{V}=\right.$ 11.39 and 11.78, respectively, Turner 1985; Kaltcheva \& Georgiev 1994). On the HR diagram of stars within $30^{\prime}$ of Stock 16, HD 115071 lies at the top of the zero-age main sequence (ZAMS), together with some of the other members of the wider Cen OB1 population, and the brightest members of the cluster proper (Turner 1985).

\section{The velocity}

Despite its brightness, and that it has been known as a radial-velocity variable for many years, HD 115071 has been particularly neglected, and still does not have a published orbit. One velocity has been published by Conti et al. (1977) and the single IUE spectrum, which reveals the system to be double lined, has been discussed repeatedly (Howarth et al. 1997; Penny 1996; Stickland \& Lloyd 2001).

Although it is clearly impossible to derive an orbit from two epochs, the velocities, particularly those from $I U E$, do provide very useful constraints on the system. The radial velocities of the components have been determined from the single IUE spectrum using a crosscorrelation technique, which has been applied in a series of papers on early-type binaries observed with IUE, 


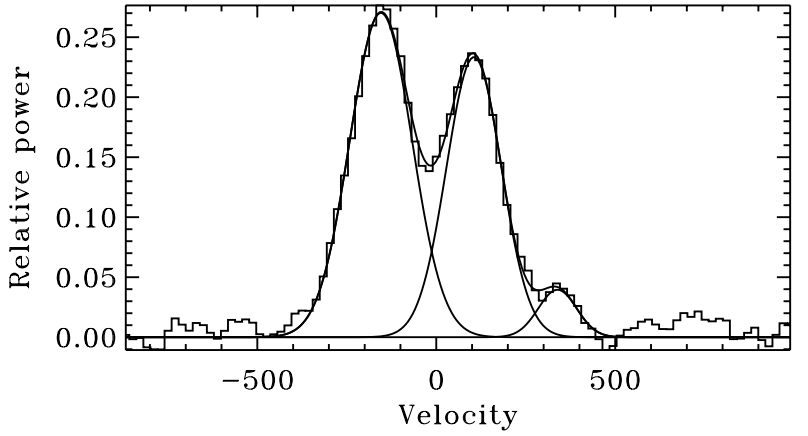

Fig. 1. Cross-correlation function of the IUE spectrum

most recently by Stickland \& Lloyd (2001). A detailed description of the method, the sections of spectrum used, and the reliability of the results is given by Stickland (1998). In essence the binary spectrum is cross correlated against a standard star of the appropriate spectral type and the velocities are determined from the peaks of the cross-correlation function $(\mathrm{CCF})$. The velocities are placed on a near-absolute footing by cross correlating the interstellar spectrum of the target star against the standard. The CCF of HD 115071 with the O9 V standard (HD 57682) is shown in Fig. 1 and two quite wellresolved components and a possible weak third component can be seen. The best correlation with the brighter component occurs with the $\mathrm{O} 9 \mathrm{~V}$ standard, and this is consistent with the spectral type given by Walborn (1973). However, for the slightly fainter component the correlation improves with standards of slightly earlier spectral type, such as HD $91824(\mathrm{O} 7 \mathrm{~V}((\mathrm{n})))$. Direct examination of the IUE spectrum suggests that both components are unevolved, late O-type stars with no obvious nitrogen enhancement. The interstellar velocity of HD 115071 is unknown but nearby stars, HD 114886 and HD 115455, have interstellar velocities from the CaII K line of $-17 \mathrm{~km} \mathrm{~s}^{-1}$ and $-19 \mathrm{~km} \mathrm{~s}^{-1}$ respectively (Crampton 1976), and the latter has been assumed to place the IUE velocities on an absolute scale. Other stars in the area observed in the $\mathrm{CH}$ and $\mathrm{CH}+$ molecular lines show similar velocities (Grendel 1997).

The systemic velocity of HD 115071 is also unknown and a value has been assumed based on the local stellar and interstellar velocities. The velocities of other stars in the area are very similar to the interstellar velocity. For example, the mean velocity of 15 stars in Cen OB1 from Humphreys (1978) is $-19 \pm 8 \mathrm{~km} \mathrm{~s}^{-1}$. A systemic velocity for HD $115071 \sim-30 \mathrm{kms}^{-1}$ would yield a mass ratio, $q \sim 1$, which seems unlikely given the difference in spectral type of the components. It is more likely to lie in the range -20 to $-10 \mathrm{~km} \mathrm{~s}^{-1}$ giving the mass ratio in the range $0.77<q<0.90$, making the hotter star (with the slightly weaker $\mathrm{CCF}$ ) the more massive of the pair.

The CCF method also provides a measure of the $v \sin i$ of the components and these, together with the radial velocities, are given in Table 1 . The values of $v \sin i$ found here are about $10 \%$ and $25 \%$ larger than
Table 1. Radial velocity measurements

\begin{tabular}{lccc}
\hline & JD & Primary & Secondary \\
Velocity $\left(\mathrm{km} \mathrm{s}^{-1}\right)$ & 2444487.4713 & +101 & -155 \\
$v \sin i\left(\mathrm{~km} \mathrm{~s}^{-1}\right)$ & & 133 & 148 \\
\hline
\end{tabular}

those of Penny (1996) and Howarth et al. (1997) respectively. All three sets show the brighter component as consistently broader than the fainter component. Conti \& Ebbets (1977) also give a value of the projected rotational velocity, presumably from the same plate that provided the velocity in the Conti et al. (1977) paper, of $v \sin i=280 \mathrm{~km} \mathrm{~s}^{-1}$. Their value is approximately twice that of the broader star because it includes the velocity separation of the components in the unresolved profile. See below and Fig. 3 .

\section{The light curve}

In the Hipparcos Catalogue (Perryman et al. 1997) HD 115071 is given as a sinusoidal variable, with $P=$ 1.36 days and a comment that it could be twice this value. For the types of stars involved it is more likely that the light curve is due to ellipsoidal variations with twice this period. A least squares fit to the data gives slightly smaller residuals than the sinusoidal fit and provides the ephemeris of primary minimum

$$
\mathrm{JD}=2448391.496( \pm 0.011)+2.73126( \pm 0.00009) \times E .
$$

The light curve has been modelled using the photometric code LIGHT2 (see Hill et al. 1989), taking as a starting point the effective temperature of the secondary, $T_{2}=$ $32000 \mathrm{~K}$, assumed from the spectral type of $\mathrm{O} 9 \mathrm{~V}$, and $q=0.90$, which is equivalent to $\gamma=-20 \mathrm{~km} \mathrm{~s}^{-1}$. The best solution, shown in Fig. 2, yields $T_{1}=39000 \pm 7000 \mathrm{~K}$ and $i=50^{\circ} \pm 9^{\circ}$. The system is an ellipsoidal variable and primary minimum occurs when the cooler, O9 V component is at inferior conjunction, i.e., in front of the hotter component. The phasing of the IUE velocities, at $\phi=0.614$ with respect to primary minimum, also places the cooler component at inferior conjunction during primary minimum. Taking the value of $q=0.90$, the $I U E$ velocities give $K_{1}=184 \mathrm{~km} \mathrm{~s}^{-1}$ and $K_{2}=206 \mathrm{~km} \mathrm{~s}^{-1}$, and radii $R_{1}=8.0 R_{\odot}$ and $R_{2}=10.0 R_{\odot}$. The radius of the hotter component is consistent with that of a late O-type mainsequence star but the cooler star is too large to be on the main sequence, and is also probably filling its Roche lobe. A circular orbit with $P=2.73$ days and $i=50^{\circ}$ gives masses, $M_{1}=19 M_{\odot}$ and $M_{2}=17 M_{\odot}$, which are consistent with the expected masses of late O-type stars, if a little low. Given the large uncertainty in the inclination the agreement is excellent.

The velocities are shown in Fig. 3, together with the orbital solutions, assuming $\gamma=-20 \mathrm{~km} \mathrm{~s}^{-1}$. The velocity from Conti et al. shows only a single, broad line and although it falls close to single-line phase on the diagram, the velocity separation of the components is $\sim 150 \mathrm{~km} \mathrm{~s}^{-1}$. 


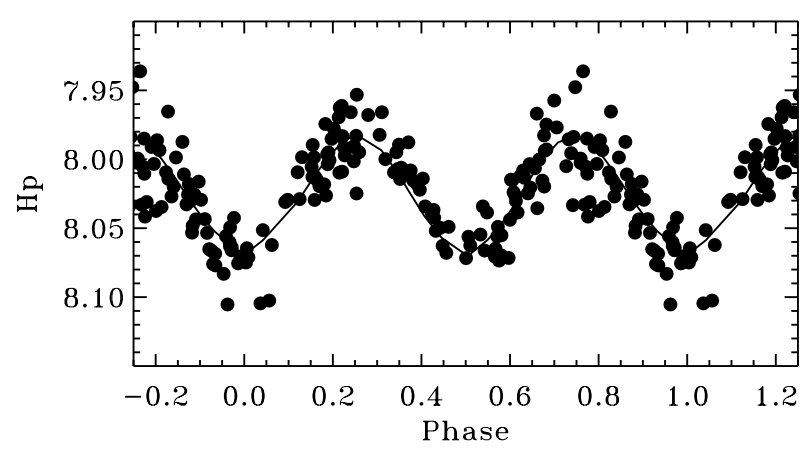

Fig. 2. The Hipparcos light curve of HD 115071 with the photometric solution over plotted

Combined with the $v \sin i$ s of the components the velocity separation would produce a single unresolved line with a width of $\sim 280 \mathrm{~km} \mathrm{~s}^{-1}$, as seen by Conti \& Ebbets.

\section{Discussion}

Both the spectroscopic and photometric evidence point to the hotter component being more massive, yet smaller and fainter than the cooler component. Despite the mainsequence classification, the relative sizes of the stars mean that it is impossible for both stars to lie on the main sequence. There are probably only two possible ways to resolve this problem. Either the cooler component is the evolved member of a mass-exchange binary or it is still contracting onto the main sequence.

The age of HD 115071 is not directly known so, until reliable stellar parameters become available that can be compared with stellar evolution models, a less direct approach will have to be adopted. It seems unlikely that HD 115071 is strictly a member of Stock 16. It lies some distance from the cluster and, being a massive binary in its own right, is unlikely to have been ejected by gravitational interactions with other cluster members. The most likely interpretation is that HD 115071 is a product of the previous generation of star formation in this region, which makes it at least as old as the brightest and most massive star in Stock 16, HD 115455, and is unlikely to have one component still contracting onto the main sequence. The position of HD 115071 at the top of the ZAMS in the HR diagram of local stars is exactly what would be expected if it were a blue straggler, belonging to a slightly older population.

While most blue stragglers have spectral types of A or F (Ahumada \& Lapasset 1995), early-type blue stragglers do occur but are comparatively rare, and approximately half of them have spectral anomalies (Mermilliod 1982). According to Monte Carlo simulations by Pols \& Marinus (1994), blue stragglers produced by mass exchange in close binaries come in five types; MS star plus helium star, white dwarf or neutron star, MS plus MS stars, or merged MS star. The main sequence pairs are produced by Case A mass transfer (Pols 1994) and this seems the most likely

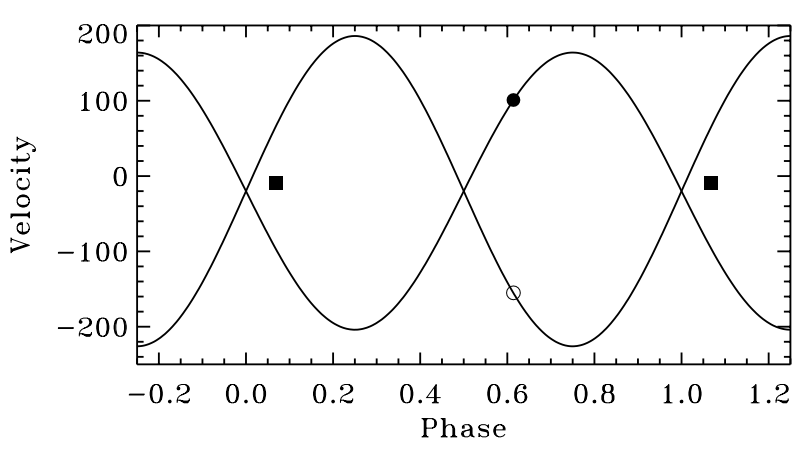

Fig. 3. The velocities of HD 115071 from Conti et al. (square) and $I U E$ (circles), with the adopted orbital solution over plotted. The phases are photometric

origin of HD 115071, although there do not appear to be any spectral anomalies.

The present mass of the system is unlikely to exceed $45 M_{\odot}$, so if one third of the mass was lost from the system during transfer, the initial total mass could have been $\sim 70 M_{\odot}$, with $M_{1}>50 M_{\odot}$ and $q<0.4$. Such a massive star would very quickly evolve into a Wolf-Rayet star, even without any mass transfer, but this has clearly not happened. Therefore, the initial mass of the primary, the total mass of the system, and probably the mass lost from the system must have been less extreme. As the system contains two apparently relatively normal stars, it suggests that mass transfer took place before the initial primary had evolved significantly, which points to Case A mass transfer. The slow growth in radius during the mainsequence evolution of the initial primary and, presumably, smooth mass transfer could have led to little mass being lost from the system.

Without detailed modelling and a reliable age it is impossible to be precise about the evolutionary stage of the system, although if the cooler star does fill its Roche lobe, mass transfer may still be occurring, or may have only recently ceased. Also, within the uncertainties, the system is probably rotationally locked. For synchronous rotation the components would have $v \sin i$ s of 113 and $142 \mathrm{~km} \mathrm{~s}^{-1}$ respectively, which are comparable with the values given in Table 1, discussed in Sect. 2.

HD 115071 lies at a similar distance to Stock 16, which is given by Turner (1985) as $1.90 \pm 0.08 \mathrm{kpc}$, with a reddening of $E_{B-V}=0.46$. Combined with the visual magnitude, and assuming $A_{V}=3.0$, this gives an absolute magnitude of $M_{V}=-4.8$ for the system. For two equal components this gives $M_{V}=-4.1$ individually, which is entirely consistent with the expected luminosity of two late O-type stars.

\section{Conclusions}

HD 115071 is an ellipsoidal variable, probably containing stars of spectral types $\mathrm{O} 7-8 \mathrm{~V}$ and O9IV. The primary is the hotter, more massive, but smaller and less luminous 
component. The system has undergone mass exchange, probably conservative Case A, and the present secondary is probably filling its Roche lobe.

\section{References}

Ahumada, J., \& Lapasset, E. 1995, A\&AS, 109, 375

Conti, P. S., \& Ebbets, D. 1977, ApJ, 213, 438

Conti, P. S., Leep, E. M., \& Loore, J. J. 1977, ApJ, 214, 759

Crampton, D. 1972, MNRAS, 158, 85

Grendel, R. 1997, A\&A, 320, 929

Hill, G., Fisher, W. A., \& Holmgren, D. 1989, A\&A, 211, 81

Howarth, I. D., Siebert, K. W., Hussain, G. A. J., \& Prinja, R. K. 1997, MNRAS, 284, 265
Humphreys, R. M. 1978, ApJS, 38, 309

Kaltcheva, N. T., \& Georgiev, L. N. 1994, MNRAS, 269, 289 Mermilliod, J. C. 1982, A\&A, 109, 37

Penny, L. R. 1996, ApJ, 463, 737

Perryman, et al. 1997, The Hipparcos and Tycho Catalogues, ESA SP-1200

Pols, O. R. 1994, A\&A, 290, 119

Pols, O. R., \& Marinus, M. 1994, A\&A, 288, 475

Stickland, D. J. 1998, in Ultraviolet Astrophysics Beyond the IUE Final Archive, Proceedings of the Conference held in Sevilla, Spain, 11-14, November, 1997, ESA SP-413, 307

Stickland, D. J., \& Lloyd, C. 2001, Observatory, 121, 1

Turner, D. G. 1985, AJ, 292, 148

Walborn, N. R. 1973, AJ, 78, 1067 